\title{
Association of the Neuronal Cell Adhesion Molecule (NrCAM) Gene Variants with Personality Traits and Addictive Symptoms in Methamphetamine Use Disorder
}

\author{
Byung Kuk Yoo', Joo Cheol Shim², Byung Dae Lee ${ }^{3,4} \bowtie$, Choongrak Kim5, Young In Chung ${ }^{3}$, Je Min Park \\ Sung Gon Kim ${ }^{3}$, Ji Hoon Kim ${ }^{3}$, Young Min Lee ${ }^{3}$, Eun Soo Moon ${ }^{3}$ and Do Hoon Kwon ${ }^{6}$ \\ ${ }^{1}$ Department of Psychiatry, Armed Force Capital Hospital, Seongnam, Republic of Korea \\ ${ }^{2}$ Department of Psychiatry, Inje Paik Hospital, Busan, Republic of Korea \\ ${ }^{3}$ Department of Psychiatry, Pusan National University Hospital, Busan, Republic of Korea \\ ${ }^{4}$ Medical Research Institute, Pusan National University Hospital, Busan, Republic of Korea \\ ${ }^{5}$ Department of Statistics, Pusan National University, Busan, Republic of Korea \\ ${ }^{6}$ Department of Psychiatry, Jindong Taebong Hospital, Changwon, Republic of Korea
}

Objective 1) To investigate the relationship between NrCAM polymorphisms and methamphetamine abuse in an ethnically homogenous Korean population. 2) To further support our findings by investigating the association among NrCAM gene variants, certain personality traits, and addictive symptoms of methamphetamine abusers.

Methods Thirty-seven male methamphetamine abusers (age $=43.3 \pm 7.8)$ and 30 non-users $(16$ men, 14 women; age $=59.8 \pm 10.4)$ were recruited. Ten single nucleotide polymorphisms (SNPs) in the NrCAM gene were assayed to compare genotype distributions between the 2 groups. Personality characteristics were measured using the Temperament and Character Inventory (TCI) and the NEO Personality Inventory, Revised (NEO PI-R). Addictive symptoms were assessed using the Diagnostic Interview for Genetic Studies (DIGS) and reviews of the subject's medical records.

Results Among the 10 SNPs in the NrCAM gene, the frequency of the TA genotype at rs1990162 was significantly lower in methamphetamine abusers compared to non-users ( $\mathrm{p}=0.042$ ). In the $3 \mathrm{NrCAM}$ gene SNPs (rs381318, rs2072546, and rs6954366), the distribution of genotypes and alleles were significantly associated with some traits in the TCI and NEO PI-R. Genotypes and alleles at 5 gene SNPs (rs2142325, rs381318, rs1269621, rs1269634, and rs1990162) were associated with certain addictive symptom dimensions in the patients.

Conclusion These findings support the idea that NrCAM is associated with genetic susceptibility of methamphetamine abuse and is also associated with certain personality characteristics that may increase disturbed addictive behavior.

Psychiatry Investig 2012;9:400-407

Key Words NrCAM, Methamphetamine abuse, Personality traits, Addictive symptoms.

\section{INTRODUCTION}

Drug abuse is a persistent global phenomenon, affecting almost every country in the world. High levels of drug dependence cause detrimental societal effects such as low pro-

Received: October 8, 2011 Revised: March 8, 2012

Accepted: May 8, 2012 Available online: November 14, 2012

$\triangle$ Correspondence: Byung Dae Lee, MD, PhD

Department of Psychiatry, Pusan National University Hospital, 305 Gudeokro, Seo-gu, Busan 602-739, Republic of Korea

Tel: +82-51-240-7304, Fax: +82-51-248-3648

E-mail: psyleebd@gmail.com

(a) This is an Open Access article distributed under the terms of the Creative Commons Attribution Non-Commercial License (http://creativecommons.org/licenses/bync/3.0) which permits unrestricted non-commercial use, distribution, and reproduction in any medium, provided the original work is properly cited. ductivity, transmission of infectious diseases, family and social disorders, increased crime, and excessive utilization of healthcare systems. ${ }^{1}$

In South Korea, statistics show that methamphetamine is the most widely abused non-prescription drug, followed by cannabis and opiates. ${ }^{2}$ Methamphetamine produces a very euphoric high, with almost instantaneous onset when the drug is smoked or injected, and excessive use is common, even in individuals with no predisposition to alcohol and drug addiction. ${ }^{3}$ Negative psychological impacts of methamphetamine usage include increased anxiety, paranoia, aggression, and violence, as well as visual and auditory hallucinations. ${ }^{4}$

Diverse and complex genetic influences have been shown 
to contribute to an individual's vulnerability toward substance abuse. ${ }^{5}$ Linkage- and association-based genome-wide studies have identified a number of chromosomal regions likely to contain variants that confer susceptibility to dependence on illegal substances. ${ }^{6}$ For example, the mid-region of human chromosome 7 has been considered a candidate site for allelic variants that are related to substance abuse and alcohol dependence. ${ }^{7}$ Convergent data from genome-wide association studies in humans and mice suggest important roles for "higher order" pharmacogenomics in the molecular genetics of addiction. ${ }^{8}$

NrCAM is a gene that encodes neuronal cell adhesion molecule, which produces a receptor involved in nervous system development expressed in the central nervous system and located in the 7q region. ${ }^{9}$ Several lines of evidence support the association of NrCAM with poly-substance abuse vulnerability in humans, as well as in the "rewarding effect" when studying abused drugs administered to animals. ${ }^{10}$

Personality traits are also predisposing factors toward addiction or an affinity toward drug usage. Drug addiction may be considered a convergence between the physiological effects of a drug and environmental factors such as one's individual personality. ${ }^{11}$ Interesting associations between certain genes, specific personality traits, and addiction symptoms have been reported in several studies. ${ }^{12,13}$

In this study, genetic polymorphisms in the NrCAM gene in South Korean methamphetamine abusers were compared with those of non-user controls. We then analyzed the associations between NrCAM gene variants and personality traits along with the addictive characteristics of methamphetamine abusers.

\section{METHODS}

\section{Subjects}

The subjects in this study included inpatients at the Inpatient Addiction Unit of the Bugok National Hospital in Korea, which has an established protocol for the evaluation, treatment, and follow-up of patients with a diagnosis of drug abuse. We identified 37 patients that fulfilled the DSM-IV diagnostic criteria for methamphetamine abuse or dependence. ${ }^{14}$ Patients also satisfied the following criteria: 1) had been methamphetamine users for more than 1 year, with the total incidence of drug use exceeding 100 times; and 2) had no known concomitant diseases that might cause any bias. Female patients were not included owing to culture-specific environmental factors.

Semi-structured interviews were performed by a psychiatrist to gather basic information such as age, marital status, and employment. Parameters for addictive behavior included the total duration of drug usage, typical dosage sizes, and the number of times arrested by police for methamphetaminerelated offenses.

Our control group consisted of 30 normal healthy volunteers with no known history of psychiatric or substance use disorders. They were also checked for an absence of major relevant medical disorders such as thyroid disorder, diabetes mellitus, etc.

Samples sizes for treatment and control were determined by power computations; for the required power 0.80 (i.e., $1-\beta=0.80$ ), we allowed the probability of type I error be 0.05 and $\Delta / \sigma=1$, where $\Delta=\mathrm{p}_{1}-\mathrm{p}_{2}$ under the alternative hypothesis, and $\sigma$ was the standard deviation of the measurement in each patient.

\section{Genotyping}

There are 10 single nucleotide polymorphisms (SNPs) present in NrCAM genes, which are functionally relevant to neuropsychiatric disorders, on chromosome 7. These include 4 exonic SNPs (rs1269621, rs2072546, rs381318, and rs6958498), 4 intronic SNPs (rs1990162, rs2142325, rs6954366, and rs722519), and 2 putative regulatory SNPs (rs1269634 and rs17155059). Genotyping was carried out by laboratory personnel who were blinded to phenotype data, as described previously by Lee et al. ${ }^{15}$ One patient was excluded from analysis because of genotyping error.

\section{Personality assessments}

We assessed personality characteristics using both Temperament and Character Inventory (TCI) and NEO Personality Inventory, Revised (NEO PI-R). ${ }^{16,17}$

The TCI is an inventory for evaluating distinct personality traits. It is closely related to the Tridimensional Personality Questionnaire (TPQ) and also derives elements from Zuckerman's and Eysenck's Dimensions of Personality. The TCI addresses 7 dimensions of personality traits with 4 so-called "temperaments" (Novelty Seeking, Harm Avoidance, Reward Dependence, and Persistence) and 3 "characters" (Self-Directedness, Cooperativeness, and Self-Transcendence). This test compliments the NEO PI-R, which is a psychological personality inventory assessment developed by Paul T. Costa Jr. and Robert R. McCrae for use with adult men and women. The NEO PI-R includes 5 major domains of personality (Neuroticism, Extraversion, Openness to Experience, Agreeableness, and Conscientiousness), as well as 6 sub-scales that further define each domain.

\section{Statistical analysis}

The genotype distribution was calculated for each individual polymorphism evaluated using Hardy-Weinberg equilib- 
rium to confirm data quality. ${ }^{18}$

The association between the case-control status and each individual SNP was measured using the odds ratio and its corresponding 95\% confidence interval using logistic regression models (adjusting for sex and age as covariates). Casecontrol analysis was conducted using 4 models. All analyses were performed assuming dominant, recessive, additive, or allelic effects for each polymorphism. In the dominant model, both the heterozygous variant and the rare homozygous variant were combined; in the recessive model, the variant was defined as the rare homozygous genotype only; in the additive model, each genotype variant had the same effect; and in the allele model, the rare allele variant had an effect. The likelihood ratio test was used to test the effect of each SNP at a
$5 \%$ significance level. All data were processed and analyzed using R software, version 2.6.2. Associations between personality dimensions or addictive symptoms and individual SNPs in abusers were then evaluated using Analysis Of Variance (ANOVA) with the SPSS system, version 11.5.

\section{RESULTS}

The demographics and clinical characteristics of methamphetamine abuse subjects are indicated in Table 1. All patients were male owing to the fact that all inpatients hospitalized in the Addiction Unit of the Bugok National Hospital were male, and females could not be recruited from elsewhere. The mean age of methamphetamine abusers was 43.3

Table 1. Demographic and clinical characteristics in methamphetamine abusers and controls

\begin{tabular}{|c|c|c|c|c|}
\hline & Characteristics & $\begin{array}{l}\text { Methamphetamine abusers } \\
\qquad \mathrm{N}=37(\%)\end{array}$ & $\begin{array}{c}\text { Controls } \\
\mathrm{N}=30(\%)\end{array}$ & $\mathrm{p}$ \\
\hline \multirow[t]{16}{*}{ Demographic } & Gender & & & \\
\hline & Male & $37(100)$ & $16(53)$ & \\
\hline & Female & - & $14(47)$ & \\
\hline & & & & 0.000 \\
\hline & Age (years) & & & \\
\hline & $<40$ & $14(38)$ & $1(3)$ & \\
\hline & $40-50$ & $15(40)$ & $7(23)$ & \\
\hline & $>50$ & $8(22)$ & $22(74)$ & \\
\hline & & $43.3 \pm 7.8$ & $59.8 \pm 10.4$ & 0.000 \\
\hline & Marital status & & & \\
\hline & Unmarried & $10(27)$ & - & \\
\hline & Married & $12(32.4)$ & - & \\
\hline & Divorced & $15(40.6)$ & - & \\
\hline & Occupation & & & \\
\hline & Unemployed & $9(24.3)$ & - & \\
\hline & Employed & $28(75.6)$ & - & \\
\hline \multirow[t]{14}{*}{ Clinical } & Duration of use (years) & & & \\
\hline & $\leq 10$ & $10(27)$ & - & \\
\hline & $10-20$ & $15(40.6)$ & - & \\
\hline & $>20$ & $12(32.4)$ & - & \\
\hline & Total dose (g) & & & \\
\hline & $<200$ & $15(40.6)$ & - & \\
\hline & $201-400$ & $13(35.1)$ & - & \\
\hline & $401-600$ & $4(10.8)$ & - & \\
\hline & $>600$ & $5(13.5)$ & - & \\
\hline & Number for arrests & & & \\
\hline & $\leq 2$ & $12(32.4)$ & - & \\
\hline & $3-4$ & $13(35.1)$ & - & \\
\hline & $5-6$ & $7(19)$ & - & \\
\hline & $>6$ & $5(13.5)$ & - & \\
\hline
\end{tabular}


\pm 7.8 years. Ten were unmarried and 15 were divorced, with over half of the subjects employed in the service industry.

The mean duration of methamphetamine abuse was 14.35 \pm 1.10 years, while the mean total dose of methamphetamine that had been consumed during the period of drug abuse was calculated to be $326.46 \pm 40.94 \mathrm{~g}$, estimated solely on the basis

Table 2. NrCAM genotype frequencies in Korean methamphetamine abusers and controls

\begin{tabular}{|c|c|c|c|c|c|c|c|c|c|c|}
\hline \multirow{2}{*}{ Loci } & \multirow{2}{*}{ Genotype } & \multicolumn{2}{|c|}{ MA } & \multicolumn{2}{|c|}{ Control } & \multirow{2}{*}{ Model } & \multirow{2}{*}{ OR } & \multicolumn{2}{|c|}{$95 \%$ CI } & \multirow{2}{*}{$\mathrm{p}$} \\
\hline & & Freq & $\%$ & Freq & $\%$ & & & LCL & UCL & \\
\hline \multirow[t]{4}{*}{ rs17155059 } & $\mathrm{AA}$ & 36 & 100 & 30 & 100 & Dominant & INF & INF & INF & INF \\
\hline & & & & & & Recessive & INF & INF & INF & INF \\
\hline & & & & & & Addictive & INF & INF & INF & INF \\
\hline & & & & & & Allele & INF & INF & INF & INF \\
\hline \multirow[t]{4}{*}{ rs2142325 } & $\mathrm{TT}$ & 33 & 91 & 29 & 96 & Dominant & 2.636 & 0.259 & 26.760 & 0.412 \\
\hline & TC & 3 & 9 & 1 & 4 & Recessive & INF & INF & INF & INF \\
\hline & $\mathrm{CC}$ & 0 & - & 0 & - & Addictive & 2.636 & 0.259 & 26.760 & 0.412 \\
\hline & & & & & & Allele & 2.565 & 0.259 & 25.323 & 0.420 \\
\hline \multirow[t]{4}{*}{ rs6958498 } & $\mathrm{CC}$ & 36 & 100 & 30 & 100 & Dominant & INF & INF & INF & INF \\
\hline & & & & & & Recessive & INF & INF & INF & INF \\
\hline & & & & & & Addictive & INF & INF & INF & INF \\
\hline & & & & & & Allele & INF & INF & INF & INF \\
\hline \multirow[t]{4}{*}{ rs381318 } & $\mathrm{AA}$ & 30 & 83 & 22 & 73 & Dominant & 0.550 & 0.166 & 1.813 & 0.326 \\
\hline & $\mathrm{AC}$ & 6 & 17 & 8 & 27 & Recessive & INF & INF & INF & INF \\
\hline & $\mathrm{CC}$ & 0 & - & 0 & - & Addictive & 0.550 & 0.166 & 1.813 & 0.326 \\
\hline & & & & & & Allele & 0.590 & 0.193 & 1.809 & 0.356 \\
\hline \multirow[t]{4}{*}{ rs1269621 } & $\mathrm{AA}$ & 26 & 72 & 20 & 66 & Dominant & 0.769 & 0.268 & 2.204 & 0.625 \\
\hline & AG & 10 & 28 & 9 & 30 & Recessive & 0.000 & 0.000 & INF & 0.991 \\
\hline & GG & 0 & - & 1 & 4 & Addictive & 0.698 & 0.262 & 1.855 & 0.471 \\
\hline & & & & & & Allele & 0.718 & 0.282 & 1.829 & 0.488 \\
\hline \multirow[t]{4}{*}{ rs2072546 } & $\mathrm{AA}$ & 12 & 33 & 9 & 30 & Dominant & 0.857 & 0.301 & 2.434 & 0.772 \\
\hline & AG & 19 & 52 & 13 & 43 & Recessive & 0.443 & 0.127 & 1.538 & 0.200 \\
\hline & GG & 5 & 25 & 8 & 27 & Addictive & 0.723 & 0.361 & 1.445 & 0.359 \\
\hline & & & & & & Allele & 0.720 & 0.361 & 1.439 & 0.353 \\
\hline \multirow[t]{4}{*}{ rs722519 } & GG & 31 & 86 & 26 & 86 & Dominant & 1.048 & 0.254 & 4.312 & 0.947 \\
\hline & AG & 5 & 14 & 2 & 7 & Recessive & 0.000 & 0.000 & INF & 0.992 \\
\hline & $\mathrm{AA}$ & 0 & - & 2 & 7 & Addictive & 0.736 & 0.247 & 2.193 & 0.582 \\
\hline & & & & & & Allele & 0.671 & 0.194 & 2.320 & 0.529 \\
\hline \multirow[t]{4}{*}{ rs6954366 } & GG & 32 & 88 & 30 & 100 & Dominant & INF & 0.0000 & INF & 0.992 \\
\hline & TG & 4 & 12 & 0 & - & Recessive & INF & INF & INF & INF \\
\hline & TT & 0 & - & 0 & - & Addictive & INF & 0.000 & INF & 0.992 \\
\hline & & & & & & Allele & INF & 0.000 & INF & 0.988 \\
\hline \multirow[t]{4}{*}{ rs1990162 } & $\mathrm{AA}$ & 13 & 36 & 7 & 23 & Dominant & 1.260 & 0.386 & 4.111 & 0.700 \\
\hline & $\mathrm{TA}$ & 16 & 44 & 19 & 63 & Recessive & 3.673 & 1.049 & 12.865 & 0.042 \\
\hline & TT & 7 & 20 & 4 & 14 & Addictive & 1.796 & 0.858 & 3.759 & 0.119 \\
\hline & & & & & & Allele & 1.711 & 0.856 & 3.416 & 0.127 \\
\hline \multirow[t]{4}{*}{ rs1269634 } & GG & 27 & 75 & 21 & 70 & Dominant & 0.777 & 0.262 & 2.303 & 0.650 \\
\hline & AG & 9 & 25 & 8 & 26 & Recessive & 0.000 & 0.000 & INF & 0.991 \\
\hline & $\mathrm{AA}$ & 0 & - & 1 & 5 & Addictive & 0.702 & 0.257 & 1.911 & 0.489 \\
\hline & & & & & & Allele & 0.714 & 0.269 & 1.891 & 0.498 \\
\hline
\end{tabular}

Freq: frequency, MA: methamphetamine abuser, OR: odds ratio, CI: confidence intervals, LCL: lower confidence limit, UCL: upper confidence limit, INF: infinite, NrCAM: neuronal cell adhesion molecule 
of the patient's statements and uncorrected for drug purity. The largest total dose of methamphetamine intake was estimated to be $1000 \mathrm{~g}$.

The mean number of arrests for methamphetamine-related offenses was $3.8 \pm 0.4$, with the highest number being 10 .

Genotype distributions at 10 NrCAM SNPs in methamphetamine abusers and non-user controls are compared in Table 2. In one SNP (rs1990162), the frequency of the TA genotype was significantly lower in the methamphetamine abusers as compared to that in controls (OR, 3.6739; 95\% CL, $1.049-12.865 ; \mathrm{p}=0.042$ ), whereas the frequencies of the AA and TT genotypes were not significantly different between the 2 groups. No significant differences in allele/genotype frequencies at the other 9 SNPs (rs1269621, rs1269634, rs2072546, rs2142325, rs381318, rs401433, rs6954366, rs6970656, and rs722519) were observed between the 2 groups.

In 3 NrCAM gene SNPs (rs381318, rs2072546, and rs 6954366), the distributions of particular genotypes or alleles were significantly associated with traits of the TCI and the NEO PI-R. Of these, rs6954366 was significantly associated with persistence $(\mathrm{p}=0.033)$ and self-transcendence $(\mathrm{p}=0.035)$ in the TCI, as well as extraversion $(\mathrm{p}=0.012)$ and friendliness $(\mathrm{p}=0.039)$ in the NEO PI-R. Significant associations were also found between rs381318 and novelty seeking ( $\mathrm{p}=0.020)$, as well as between $\mathrm{rs} 2072546$ and reward dependence $(\mathrm{p}=0.420)$ (Table 3).

In addition, genotypes or alleles at 5 of the SNPs (rs2142325, rs381318, rs1269621, rs1990162, and rs1269634) were associated with certain addictive characteristics. Of these, rs2142325 was associated with 3 addictive characteristics, including duration of abuse $(\mathrm{p}=0.025)$, total dose $(\mathrm{p}=0.046)$, and the number of arrests ( $p=0.038)$; rs1269634 was significantly associat- ed with total dose $(\mathrm{p}=0.049)$ and the number of arrests $(\mathrm{p}=$ $0.023)$; and $\mathrm{rs} 381318$ ( $\mathrm{p}=0.053), \mathrm{rs} 1269621$ ( $\mathrm{p}=0.013)$, and $\mathrm{rs}$ $1990162(\mathrm{p}=0.056)$ were significantly associated with the number of arrests. One SNP (rs381318) was also associated with both personality and addictive symptom dimensions (Table 4).

For multiple comparisons, we used the false discovery rate (FDR) instead of the family-wise error rate (FWER), such as Bonferroni correction. FWER controls the probability of at least 1 false positive, and FDR controls the expected value of false positives in $\mathrm{m}$ simultaneous hypotheses. To be specific, we let $p_{1} \leq p_{2} \leq \cdots \leq p_{m}$ be ordered by $\mathrm{p}$-values of $\mathrm{m}$ simultaneous hypotheses with a given $q$ value. Then, we rejected $\mathrm{j}$ hypotheses corresponding to $p_{1} \leq p_{2} \leq \cdots \leq p_{i}$ where $j$ satisfied $j=\max 1 \leq i \leq n\left\{i \mid p_{i} \leq i_{q} / m\right\}$. By letting $q=0.2$, the following results occurred.

Table 2. 1) dominant model: no one is significant. 2) recessive model: rs1990162 is significant. 3) additive model: no one is significant. 4) allele model: no one is significant.

Table 3. rs381318 is significant in novelty seeking (NS) of TCI.

Table 4. rs2142325 is significant in duration of use (DU) of addictive symptoms and rs1269621 is significant in number for arrests by police (NA) of addictive symptoms.

\section{DISCUSSION}

The NrCAM gene encodes a neuronal cell adhesion molecule with multiple immunoglobulin-like C2-type domains and fibronectin type III domains. ${ }^{19} \mathrm{NrCAM}$ is an ankyrinbinding protein involved in neuron-neuron adhesion and promotes directional signaling during axonal cone growth. It is also expressed in non-neural tissues and may play a gener-

Table 3. Association analysis between NrCAM gene SNP genotypes and alleles and personality traits in methamphetamine abusers

\begin{tabular}{|c|c|c|c|c|c|c|c|c|c|c|c|c|c|}
\hline \multirow{2}{*}{ SNP_Name } & \multirow{2}{*}{ Location } & \multicolumn{7}{|c|}{$\mathrm{TCI}$} & \multicolumn{5}{|c|}{ NEO PI - R } \\
\hline & & NS & HA & $\mathrm{RD}$ & Per & $\mathrm{SD}$ & $\mathrm{Coo}$ & ST & Ex & $\mathrm{A}$ & $\mathrm{C}$ & $\mathrm{Ne}$ & $\mathrm{O}$ \\
\hline rs17155059 & UTR-3 & ns & ns & ns & ns & ns & ns & ns & ns & ns & ns & ns & ns \\
\hline rs2142325 & Intron & ns & ns & ns & ns & ns & ns & ns & ns & ns & ns & ns & ns \\
\hline rs6958498 & Exon & ns & ns & ns & ns & ns & ns & ns & ns & ns & ns & ns & ns \\
\hline rs381318 & Exon & 0.02 & ns & ns & ns & ns & ns & ns & ns & ns & ns & ns & ns \\
\hline rs1269621 & Exon & ns & ns & ns & ns & ns & ns & ns & ns & ns & ns & ns & ns \\
\hline rs2072546 & Exon & ns & ns & 0.042 & ns & ns & ns & ns & ns & ns & ns & ns & ns \\
\hline rs722519 & Intron & ns & ns & ns & ns & ns & ns & ns & ns & ns & ns & ns & ns \\
\hline rs6954366 & Intron & ns & ns & ns & 0.033 & ns & ns & 0.035 & 0.012 & 0.039 & ns & ns & ns \\
\hline rs1990162 & Intron & ns & ns & ns & ns & ns & ns & ns & ns & ns & ns & ns & ns \\
\hline rs1269634 & UTR-5 & ns & ns & ns & ns & ns & ns & ns & ns & ns & ns & ns & ns \\
\hline
\end{tabular}

TCI: Temperament \& Character Inventory, NEO PI-R: Revised NEO Personality Inventory, NS: novelty seeking, HA: harm avoidance, RD: reward dependence, Per: persistence, SD: self-directiveness, Coo: cooperativeness, ST: self-transcendence, Ex: extraversion, A: agreeableness, C: conscientiousness, Ne: neuroticism, O: openness to experience, ns: non-significant, NrCAM: neuronal cell adhesion molecule, SNP: single nucleotide polymorphism 
Table 4. Association analysis between NrCAM gene SNP genotypes and alleles and addictive symptoms in methamphetamine abusers

\begin{tabular}{lcccc}
\hline \multirow{2}{*}{ SNP_Name } & \multirow{2}{*}{ Location } & \multicolumn{3}{c}{ Addictive symptom } \\
\cline { 3 - 5 } & & DU & TD & NA \\
\hline rs17155059 & UTR-3 & ns & ns & ns \\
rs2142325 & intron & 0.025 & 0.046 & 0.038 \\
rs6958498 & exon & ns & ns & ns \\
rs381318 & exon & ns & ns & 0.053 \\
rs1269621 & exon & ns & ns & 0.013 \\
rs2072546 & exon & ns & ns & ns \\
rs722519 & intron & ns & ns & ns \\
rs6954366 & intron & ns & ns & ns \\
rs1990162 & intron & ns & ns & 0.056 \\
rs1269634 & UTR-5 & ns & 0.049 & 0.023 \\
\hline
\end{tabular}

DU: duration of use, TD: total dose, NA: number for arrests by police, ns: non-significant, NrCAM: neuronal cell adhesion molecule, SNP: single nucleotide polymorphism

al role in cell-to-cell communication via signaling from its intracellular domain to the actin cytoskeleton during directional cell migration. Allelic variants of this gene have been associated with psychiatric disorders such as autism and addiction..$^{20,21}$ Recently, NrCAM polymorphisms and haplotypes have been associated to an increased risk of substance abuse, possibly by affecting expression levels of NrCAM in the brain. In addition, some of these polymorphisms and haplotypes have been associated with altered NrCAM expression in human postmortem brain samples. ${ }^{9}$ Although potentially important neurobiological roles of $\mathrm{NrCAM}$ have been reported in animal studies, ${ }^{9,10}$ few studies have focused on human subjects.

We assayed 10 NrCAM gene SNPs to determine the association between these changes and methamphetamine abuse in 37 male methamphetamine abusers. The frequency of the TA genotype in 1 SNP (rs1990162) in methamphetamine abusers was significantly lower as compared to that in the controls ( $\mathrm{p}=0.042$ ), while no significant differences were found at any of the other 9 SNPs present in the NrCAM gene. These observations lend some support to our hypothesis that $\mathrm{NrCAM}$ is a methamphetamine abuse-associated gene with variants that affect substance abuse predisposition in human subjects. However, our results could also be considered insignificant, if more stringently interpreted. However, several issues need to be addressed in the future. Validation studies on the 10 SNPs of NrCAM need to be performed to compliment our limited information on the association between the NrCAM gene and methamphetamine abuse. Ethnic differences also need to be taken into consideration. The phases of addiction vulnerability association of the $30 \mathrm{NrCAM} 520$ Pro/Ala710c/ p1343a 3'UTR haplotype was similar in 2 previous European-American substance abuser/control comparisons but this was found to be opposite in the study on African-American subjects. ${ }^{9}$ Our study focused on an ethnically homogenous Korean population, and therefore our results relating to the NrCAM gene may only be partially relevant to other ethnic groups. It is very critical, but very difficult to recruit sufficient numbers of well-matched cases and controls with respect to age and sex for case-control association analysis. Given that our case and control subjects were poorly matched, we also analyzed our data after adjusting for age and sex for the results to be negative (rs1990162) although not recruiting matched control in this study due to the very many limitations. We are now in the process of recruiting a larger cohort of matched control samples to further confirm the findings of the present study. However, it should be noted that current opinion in the field is that in some cases it is sufficient to conduct case-control association studies without age-sex adjustment, despite possible issues concerning population stratification and related confounding effects.

Molecular heterosis also deserves a mention; this occurs when heterozygous genotypes at a locus exhibit significantly larger (positive heterosis) or smaller (negative heterosis) effects on a quantitative or dichotomous trait than homozygous genotypes. There is accumulating evidence that molecular heterosis is common in humans and may occur in up to $50 \%$ of all genetic associations. Three explanations for molecular heterosis are proposed. The first is based on an inverted Ushaped response curve in which either too little or too much gene expression is deleterious; in this case, optimal gene expression would be associated with heterozygous genotypes. The second proposes an independent third factor causing a hidden stratification of the sample such that in 1 set of subjects homozygosity is associated with the highest phenotype score, while in the other set, homozygosity is associated with the highest phenotype score. The third explanation suggests greater fitness in heterozygotes because they show a broader range of gene expression than homozygotes. Allele-based linkage techniques usually neglect heterotic associations. Because up to $50 \%$ of association studies show a heterosis effect, this can significantly diminish the power of family-based linkage and association studies. This point could be complemented by 4 different genetic model analyses (dominant, recessive, additive, and allelic), such as those shown in Table 2. In some cases it could also be speculated that differences between the AT genotype (heterozygote) and the AA and TT genotypes (homozygotes) are the result of epigenetic factors. ${ }^{22}$

Several previous studies have reported personality differences between addicts and normal subjects. ${ }^{23}$ These have highlighted a correlation between behavioral disorders, such as hy- 
peractivity and later-life drug consumption. It is common knowledge that certain individuals are more susceptible to partake in risky activities, such as drug abuse, whereas others are more inclined to avoid them. The neurological mechanism of drug addiction is often pathological and may share features in common with psychotics, pre-psychotics, and psychopaths, with the physiological effects of drugs playing an important role in decompression in these personality types. ${ }^{11}$

Our finding of an association between the NrCAM gene and addictive symptoms in methamphetamine abusers supports the model that addiction vulnerability may be strongly influenced by a genetic predisposition. ${ }^{24,25}$ In our association analysis between the NrCAM SNP genotypes and personality traits in methamphetamine abusers, we found that noveltyseeking traits were associated with rs381318, while rewarddependence traits were associated with rs2072586. In addition, the SNP rs6954366 was found to be significantly associated with persistence, self-transcendence, extraversion, and agreeableness traits. Further studies need to be performed with welldefined scales for personality assessment and a larger sample size.

After investigation of the relationship between the NrCAM gene and addictive symptoms, 5 SNPs (rs2142325, rs381318, rs1269621, rs1269634, and rs1990162) were found to be associated with addictive symptoms. In particular, rs2142325 was significantly associated with all defined addiction parameters (duration of drug use, total dose, and number of arrests). rs1269634 was also significantly associated with total dose and number of arrests, while rs381318, rs1269621, and rs1990162 were only significantly associated with the number of arrests. Although we found some associations between the NrCAM gene and addictive symptoms, the clinical significance of these findings needs to be confirmed in future studies.

This study has several limitations. First, given that we used a small sample size, the statistical power of our analyses was reduced, and thus, further studies, using larger study cohorts than those used in our study, should be undertaken. The second limitation was that demographic characteristics such as gender and age were not matched between methamphetamine abusers and controls. The third is that the 10 SNPs of NrCAM gene were not validated by assaying. In addition, the scale for measures of personality dimension and the criteria of addictive symptoms were broad, and could be more clearly refined. Finally, and most importantly, unlike other psychiatric disorders that are primarily caused by underlying etiologic factors, addiction is a disease that occurs only in people who have been exposed to a substance. Therefore, it is very difficult, but important to control for both environmental factors that are crucial in the onset of addiction, as well as genetic factors (i.e., an individual who is not addicted despite having been exposed to methamphetamine). Thus, the difference between patients and controls could simply be the result of different levels of exposure to methamphetamine; however, it is important to note that genetic factors can also dictate patterns of behavior that result in greater exposure to risk stimuli.

Future research should place emphasis on denser genotyping panels, sequencing, and functional studies. These may help to confirm whether NrCAM or other genes in linkage disequilibrium with this candidate, contain causal variants that influence the pathogenesis of addiction in Korean and other ethnic populations.

\section{Acknowledgments}

This study was supported by Biomedical Research Institute Grant (200930), Pusan National University Hospital.

\section{REFERENCES}

1. Kulsudjarit K. Drug problem in southeast and southwest Asia. Ann N Y Acad Sci 2004; 1025:446-457.

2. Chung H, Park M, Hahn E, Choi H, Choi H, Lim M. Recent trends of drug abuse and drug-associated deaths in Korea. Ann N Y Acad Sci 2004;1025:458-464.

3. Matsumoto T, Kamijo A, Miyakawa T, Endo K, Yabana T, Kishimoto $\mathrm{H}$, et al. Methamphetamine in Japan: the consequences of methamphetamine abuse as a function of route of administration. Addiction 2002;97:809-817.

4. Murray JB. Psychophysiological aspects of amphetamine-methamphetamine abuse. J Psychol 1998;132:227-237.

5. Kendler KS, Karkowski LM, Neale MC, Prescott CA. Illicit Psychoactive substance use, heavy use, abuse and dependence in a US population-based sample of male twins. Arch Gen Psychiatry 2000;57:261-269.

6. Uhl GR. Molecular genetics of substance abuse vulnerability: remarkable recent convergence of genome scan results. Ann N Y Acad Sci 2004; 1025:1-13.

7. Uhl GR, Liu QR, Naiman D. Substance abuse vulnerability loci: converging genome scanning data. Trends Genet 2002;18:420-425.

8. Uhl GR, Drgon T, Johnson C, Fatusin OO, Liu QR, Contoreggi C, et al. "Higher order" addiction molecular genetics: convergent data from genome-wide association in humans and mice. Biochem Pharmacol 2008;75:98-111.

9. Ishiguro H, Liu QR, Gong JP, Hall FS, Ujike H, Morales M, et al. NrCAM in addiction vulnerability: positional cloning, drug-regulation, haplotype-specific expression, and altered drug reward in knockout mice. Neuropsychophamacology 2006;31:572-584.

10. Uhl GR. Molecular genetics of addiction vulnerability. NeuroRx 2006;3: 295-301.

11. Rosselli M, Ardila A, Lubomski M, Murray S, King K. Personality profile and neuropsychological test performance in chronic cocaine-abusers. Int J Neurosci 2001;110:55-72.

12. Wu CY, Wu YS, Lee JF, Huang SY, YU L, Ko HC, et al. The association between DRD2/ANKK1, 5-HTTLPR gene, and specific personality trait on antisocial alcoholism among Han Chinese in Taiwan. Am J Med Genet B Neuropsychiatr Genet 2008;147B:447-453.

13. Wang TJ, Huang SY, Lin WW, Lo HY, Wu PL, Wang YS, et al. Possible interaction between MAOA and DRD2 genes associated with antisocial alcoholism among Han Chinese men in Taiwan. Prog Neuropsychophamacol Biol Psychiatry 2007;19;31:108-114.

14. American Psychiatric Association. Diagnostic and Statistical Manual of Mental Disorders. Washington D.C.: American Psychiatric Publish- 
ing;1996.

15. Lee BD, Walss-Bass C, Thompson PM, Dassori A, Mentoro PA, Medina $\mathrm{R}$, et al. Malic enzyme 2 and susceptibility to psychosis and mania. Psychiatry Res 2007;150:1-11.

16. Cloninger CR. A practical way to diagnosis personality disorder: a proposal. J Pers Disord 2000;14:99-108.

17. Conner KR, Zhong Y, Duberstein PR. NEO PI-R neuroticism scores in Substance-dependent outpatients: internal consistency and selfpartner agreement. J Pers Assess 2004;83:75-77.

18. Weir BS. Genetic Data Analysis II : Methods for Discrete Population Genetic Data. Sunderland, MA: Sinauer Associates; 1996.

19. Lane RP, Chen XN, Yamakawa K, Vielmetter J, Korenberg JR, Dreyer WJ. Characterization of a highly conserved human homolog to the chicken neural cell surface protein Bravo/Nr-CAM that maps to chromosome band 7q31. Genomics 1996;35:456-465.

20. Dry K, Kenwrick S, Rosenthal A, Platzer M. The complete sequence of the human locus for NrCAM-related cell adhesion molecule reveals a novel alternative exon in chick and man and conserved genomic organization for the L1 subfamily. Gene 2001;273:115-122.

21. Marui T, Funatogawa I, Koishi S, Yamamoto K, Matsumoto H, Hashimoto O, et al. Association of the neuronal cell adhesion molecule (NrCAM) gene variants with autism. Int J Neuropsychophamacol 2009;12: $1-10$.

22. Comings DE, MacMurray JP. Molecular heterosis: a review. Mol Genet Metab 2000;71:19-31.

23. Nathan PE. The addictive personality is the behavior of the addict. J Consult Clin Psychol 1988;56:183-188.

24. Uhl GR, Drgon T, Johnson C, Liu QR. Addiction genetics and pleiotropic effects of common haplotypes that make polygenic contributions to vulnerability to substance dependence. J Neurogenet 2009;23: 272-282.

25. Cloninger CR. A systematic method for clinical description and classification of personality variants. A proposal. Arch Gen Psychiatry 1987; 44:573-588. 